\title{
REIGNY, Cartulaire du XIVe siècle
}

Le cartulaire de l'abbaye cistercienne de Reigny. Un recueil daté de la fin du XIV ${ }^{\mathrm{e}}$ siècle

\section{Marlène Hélias-Baron}

\section{(2) OpenEdition}

Journals

Édition électronique

URL : https://journals.openedition.org/cem/2532

DOI : $10.4000 /$ cem. 2532

ISSN : 1954-3093

Éditeur

Centre d'études médiévales Saint-Germain d'Auxerre

\section{Référence électronique}

Marlène Hélias-Baron, «REIGNY, Cartulaire du XIVe siècle », Bulletin du centre d'études médiévales d'Auxerre I BUCEMA [En ligne], Collection CBMA, mis en ligne le 25 septembre 2007, consulté le 24 septembre 2022. URL : http://journals.openedition.org/cem/2532; DOI : https://doi.org/10.4000/cem. 2532

Ce document a été généré automatiquement le 24 septembre 2022.

\section{cc) (†) (2)}

Creative Commons - Attribution - Pas d'Utilisation Commerciale - Partage dans les Mêmes Conditions 4.0 International - CC BY-NC-SA 4.0

https://creativecommons.org/licenses/by-nc-sa/4.0/ 


\section{REIGNY, Cartulaire du XIVe siècle}

Le cartulaire de l'abbaye cistercienne de Reigny. Un recueil daté de la fin du XIV ${ }^{\mathrm{e}}$ siècle

\section{Marlène Hélias-Baron}

1 L'abbaye de Reigny, installée dans le diocèse d'Auxerre, est caractéristique du renouveau monastique de la fin du $\mathrm{XI}^{\mathrm{e}}$ siècle et du début du siècle suivant. Aux origines, c'était un petit ermitage construit en 1104 au lieu-dit Fontemoy - c'est-à-dire Fons humidus - dans le diocèse d'Autun ${ }^{1}$. A la fin des années 1120, comme de nombreux autres établissements érémitiques en difficulté, il s'affilie à l'ordre de Cîteaux par l'intermédiaire de Clairvaux. En 1134, sous la direction du nouvel abbé, Etienne de Toucy, envoyé par Bernard, les moines changent à la fois de site et de diocèse en s'installant à Reigny sur les bords de la Cure, non loin de la route reliant Vermenton à Vézelay $^{2}$. Le jeune monastère devient de ce fait la cinquième fille de Clairvaux. Il n'a fait aucun essaimage, ce qui ne laisse pas de surprendre. En fait, le nouvel établissement attire très vite les largesses des seigneurs, chevaliers ou simples laïcs installés aux environs, ce qui permet à la communauté - fort nombreuse - de vivre correctement sans avoir besoin d'envoyer au loin ses moines surnuméraires. Par conséquent, les actes consignant ces donations sont de plus en plus nombreux à mesure que le XII ${ }^{\mathrm{e}}$ siècle avance. Le siècle suivant apparaît comme la période la plus faste - c'est le moment où la construction du réfectoire est entreprise - et la mieux fournie en chartes, même si de nombreux titres délivrés aux $\mathrm{XIV}^{\mathrm{e}}$ et $\mathrm{XV}^{\mathrm{e}}$ siècles témoignent encore du rayonnement de l'abbaye. Dans ces conditions, son chartrier est un des plus riches des Archives départementales de l'Yonne ${ }^{3}$. La majorité des documents encore disponibles se trouve à Auxerre, à l'exception de quelques actes présents aux Archives nationales ${ }^{4}$, et surtout du cartulaire conservé à la Bibliothèque nationale sous la cote ms. lat. $17725^{5}$. C'est cet ouvrage qui nous intéresse ici, non seulement pour son contenu, somme toute assez traditionnel, mais aussi pour les circonstances de sa fabrication ${ }^{6}$.

Présentation du cartulaire de Reigny

2 Le cartulaire de Reigny compte 37 feuillets-papier en très mauvais état, récapitulant une cinquantaine d'actes délivrés entre 1147 et 1405. Il ne comporte aucune préface, comme la majorité des cartulaires cisterciens, qui ne sont que des documents de 
gestion interne. Les noms du commanditaire et des copistes, comme les circonstances de sa rédaction, restent inconnus, même si l'abbé n'est sûrement pas resté étranger à l'entreprise ${ }^{7}$. Il ne comporte aucune table récapitulative de son contenu. En marge toutefois apparaissent quelques titres laconiques contemporains de la rédaction de l'ouvrage et des analyses plus détaillées datant de l'époque moderne, probablement de la main qui fit une première copie du cartulaire au cours du XVIII siècle ${ }^{8}$.

Ce recueil a été conservé avec le reste des archives à l'abbaye, qui subit des inondations fréquentes à partir de l'époque moderne, dues au flottage des troncs d'arbre dans la Cure. Ces derniers avaient tendance à bloquer la rivière et à dégrader le pertuis de Reigny, ce qui faisait monter les eaux jusqu'à l'église et au cloître ${ }^{9}$. Les actes et les livres conservés dans l'armarium du rez-de-chaussée ont été endommagés par l'humiditée ${ }^{10}$. C'est pourquoi, plusieurs folios du cartulaire sont presque illisibles, en particulier les trois premières feuilles, puis les dernières à partir du folio 27 et jusqu'au folio 29. Les pages abîmées sont celles qui étaient en contact avec la couverture. Toutes les marges extérieures sont rongées. Pour lire ce recueil entièrement, le recours aux originaux et aux copies d'érudits ultérieures s'avère indispensable ${ }^{11}$. Dans ce cas pourtant, le premier acte et les cinq derniers restent encore - pour l'instant - très difficiles à identifier.

Analyse codicologique

4 L'analyse codicologique n'est pas aisée à faire tant l'état du volume est mauvais; le cartulaire a en plus été entièrement réparé au XIX siècle. Les cahiers d'origine - s'il y en avait - n'ont pas été conservés; il ne reste à présent que des feuillets séparés, probablement rassemblés dans leur ordre d'origine, mais rien n'est moins sûr. La couverture est typique des restaurations de la Bibliothèque nationale de la fin du $\mathrm{XIX}^{\mathrm{e}}$ ou du début du $\mathrm{XX}^{\mathrm{e}}$ siècle.

5 Quelques indications archéologiques sur le document peuvent pourtant être glanées. Le codex mesure actuellement $22 \mathrm{~cm}$ de large sur $29 \mathrm{~cm}$ de haut. Les feuillets sont paginés de 1 à 37 par deux mains : une première, à l'époque moderne, a numéroté de 1 à 35 , et une seconde, de 36 à $37^{12}$. De nombreuses feuilles ont été laissées vierges à la fin, probablement pour ajouter ultérieurement des actes et un titre a été ajouté en toute fin au cours du XVI ${ }^{e}$ siècle $^{13}$. Les documents n'ont pas toujours été transcrits les uns à la suite des autres : des espaces vides apparaissent au bas de nombreuses pages ${ }^{14}$, certains ayant été comblés par des ajouts ultérieurs. A la lecture des actes, le manque de cohérence interne est flagrant comme si les copistes les avaient copiés sur des feuillets volants, parfois recto-verso, avant de les rassembler en un unique recueil, mais rien de permet de confirmer ou d'infirmer cette hypothèse.

Les mains

6 Cette œuvre est le fruit d'un travail collectif, mais semble souffrir de l'absence d'un véritable concepteur, d'un "cartulariste». Au moins deux scribes sont intervenus de manière relativement anarchique. L'écriture du premier copiste (scribe A) se caractérise par des lettres plus larges, la présence de point dans les panses des majuscules et l'emploi d'une encre plus claire, souvent ocre. Son acte le plus récent date de 1381. Le deuxième intervenant (scribe B) a une écriture serrée, souvent peu lisible; il utilise une encre noire ou brun foncé. Son acte le plus récent date de 1405. Enfin, un dernier copiste a ajouté au XVI ${ }^{e}$ siècle un ultime acte, mais son intervention unique - n'est pas significative. 
7 Dans ces conditions, on peut affirmer que la transcription du cartulaire a été faite à la fin du XIV ${ }^{\mathrm{e}}$ ou au tout début du XV siècle.

8 Sur ce cartulaire, n'ont été ajoutées aucune décoration, aucune lettre ornée ni aucune couleur. L'ensemble est très sobre et donne l'impression que le travail a été rapide, voire bâclé. Il n'y a en effet aucune table des matières, aucun titre en rubrique, ni aucune analyse contemporaine au dessus des actes. Ces derniers ont été copiés sur papier et non sur parchemin : l'édition est loin d'être un produit de luxe. En outre, peu de documents ont été sélectionnés, ce qui laisse supposer que la volonté des scribes n'était pas de faire un recueil exhaustif des chartes de l'abbaye. Ils avaient une autre idée en tête.

Classement

9 Le classement n'est pas apparent, du moins il ne semble pas fondé sur les granges de l'abbaye contrairement à nombre de cartulaires cisterciens ${ }^{15}$. Un embryon d'organisation se dessine néanmoins: les huit premières chartes sont les plus anciennes, elles ont toutes été délivrées avant 1150. Puis viennent d'autres documents sans ordre chronologique véritable traitant toujours de possessions groupées autour de la grange abbatiale. L'acte 14 apparaît comme un jalon. Daté de 1248 , il est la confirmation par le seigneur de Noyers de tous les dons faits sans son consentement ou celui de ces ancêtres par ses vassaux ou leurs aïeuls aux moines de Reigny ${ }^{16}$. De ce fait, il pourrait clôturer de manière logique une première partie. En effet, à partir de l'acte 15 et jusqu'à l'acte 24 , les documents s'intéressent plus particulièrement aux eaux de la Cure, à la pêcherie de l'abbaye et à ses droits de pêche dans la rivière attenante. Puis, à partir de l'acte 25 , il n'y a de nouveau plus d'ordre apparent: sont mélangés des documents traitant de vignes situées à Beaumont, de rentes ou de cens, récapitulant ou accordant des droits de justice, consignant des interdictions de prendre aux moines et à leurs hommes leurs chevaux et leurs chariots, ainsi que quelques jugements... le tout concernant exclusivement des biens situés aux alentours de l'abbaye, jamais sur une autre grange.

10 Finalement, un classement précis ne s'imposait pas pour ce cartulaire qui ne contient que des chartes et notices s'intéressant aux possessions dépendant directement de la grange de Reigny. Les biens énumérés sont tous circonscrits dans un espace compris entre les terres de l'abbaye de Vézelay et la ville de Vermenton, le long de la Cure généralement. Quelles peuvent en être les raisons?

Le cartulaire dans l'histoire de l'abbaye

11 Le cartulaire a été fabriqué après la fin des troubles liés à la guerre de cent ans. Deux de ses chartes traitent explicitement des difficultés des moines pendant cette période. Dans la première, en 1355, Jean de Chalon, comte d'Auxerre, seigneur de Rochefort et bouteiller de France, donne l'autorisation aux moines de creuser un fossé devant la porte de leur abbaye et d'y mettre un pont-levis pour se protéger des attaques éventuelles ${ }^{17}$. Le comte était coutumier du fait, puisqu'il accorda au village de Chitry la permission de fortifier l'église paroissiale en $1364^{18}$. En fait, les pouvoirs publics - rois, ducs, comtes, voire simples seigneurs - ont encouragé ou provoqué la mise en défense des villages et des monastères isolés, cibles privilégiées des pilleurs ${ }^{19}$.

12 En 1378, Charles V délivre aux moines un diplôme leur donnant le droit de se réfugier à Vermenton en cas de problème, malgré l'opposition des habitants de ladite ville ${ }^{20}$. La mise à disposition d'un lieu où se réfugier si le besoin s'en faisait sentir est une obligation du seigneur ${ }^{21}$. Ce service n'est pas gratuit puisque les moines ont dû 
contribuer à la réparation des remparts et ont dû faire construire à leurs frais à l'intérieur de l'enceinte une maison pour s'y réfugier. Ce privilège royal n'est pas à dédaigner et répond à une crainte tout à fait fondée. En effet, selon une charte datée de septembre 1369, l'abbaye et ses environs ont déjà été dévastés lors du passage d'hommes d'armes en Auxerrois ${ }^{22}$. Le bétail a été pris ou tué, l'abbaye et sa grange incendiées; tout ce que les moines avaient apporté avec eux à Vermenton lors de leur fuite a été saisi après la prise de la ville, l'abbé devant même payer une substantielle rançon pour sa libération ${ }^{23}$. Les moines se retrouvaient dans une situation si critique que l'abbé et la major et sanior pars totius conventus ont dû demander aux abbés cisterciens réunis à Cîteaux la permission d'aliéner une partie de leurs biens pour restaurer les bâtiments endommagés ${ }^{24}$. En fait, ils sollicitaient le droit de faire une coupe de 100 arpents dans les bois de Tréclin à la fois pour les réparations et pour le remboursements des dettes contractées auprès de seigneurs et autres personnes compatissantes qui leur avaient prêté du blé, du vin et de l'argent pour les soutenir quelques temps. Un rapport d'inspection fut établi peu de temps après par les abbés de Quincy et des Echarlis, commissaires délégués par le chapitre général, pour constater la nécessité, mais aussi l'urgence de cette coupe ${ }^{25}$. En 1371 enfin, par un acte attaché au rapport précédent, les abbés de Clairvaux et de Fontenay ont donné l'autorisation de procéder à la coupe pour éteindre les dettes ${ }^{26}$.

13 Dans ces conditions, une fois la paix revenue, il fallait réaffirmer les droits du monastère et protéger l'ensemble des biens relevant de la grange abbatiale en partie détruite. Ainsi, le cartulaire participe de cette reprise en main: les actes copiés ne concernent en effet que le domaine de Reigny, les droits de pêche pesant sur la Cure toute proche, les droits de justice et les cens pesant sur les terres abbatiales entre Reigny et Vermenton. Pourtant, malgré cet effort de réorganisation, les séquelles du passage des Grandes compagnies se sont fait sentir encore longtemps. En effet, les moines de Reigny ont été dans l'obligation de demander des allègements de rentes à divers seigneurs et établissements religieux de la région. Par exemple, ils devaient aux bénédictines de Crisenon une rente de dix-huit bichets de froment et de 4 livres tournois sur les moulins d'Arcy. Ceux-ci ont été détruits. En 1364, une première transaction a été envisagée pour échelonner le paiement de la rente ${ }^{27}$. En 1452, la situation ne s'était pas améliorée et les moines n'avaient pas réussi à acquitter leurs dettes : une nouvelle réunion a alors été nécessaire entre les deux communautés pour tenter de résoudre ce problème ${ }^{28}$.

La copie du cartulaire et les éditions des actes de Reigny

14 Le cartulaire médiéval est connu par deux copies manuscrites. Il a d'abord été copié en partie au cours du XVIII ${ }^{e}$ siècle. Le copiste a rencontré de nombreuses difficultés pour le transcrire: le recueil original était sans doute déjà très abîmé et peu lisible par endroits ${ }^{29}$. Au début du XX siècle, le comte de Chastellux en a fait une nouvelle copie ${ }^{30}$. Son travail n'a pas été édité mais est resté sous une forme manuscrite ; il est conservé aux Archives départementales de l'Yonne, sous la cote H 1562-1. L'érudit a laissé luiaussi de nombreux blancs dans sa transcription qui correspondent aux trous de la précédente copie, comme s'il s'en était inspiré et avait laissé de côté le cartulaire médiéval; cette hypothèse est renforcée par le fait qu'il est habituellement très consciencieux dans son travail de copie des titres de l'abbaye ${ }^{31}$.

15 Par ailleurs, outre la Gallia christiana toujours pratique, une grande partie des actes de Reigny est connue soit par les travaux de l'abbé Lebeuf, soit par l'œuvre de Maximilien 
Quantin, à savoir le fameux Cartulaire général de l'Yonne ${ }^{32}$. Outre cela, une charte rédigée en français et datée de 1164 a fait l'objet de deux articles simultanés en 1973 dans la $B E C^{33}$. Tout le problème tourne autour de l'emploi du français à une date si précoce. Le premier auteur, Denis Escudier, qui édite dans le même temps le document incriminé au début de son article, considère qu'il s'agit d'un faux forgé entre 1247 et $1282^{34}$. Pour Louis Carolus-Barré, son contradicteur, il s'agit non d'un faux, mais d'une traduction en français d'un acte authentique en latin ${ }^{35}$. Plus récemment, Nathalie Cêtre, en annexe de son mémoire de maîtrise, a reproduit quelques actes déjà édités ${ }^{36}$.

Les mentions éparses sur les chartes de Reigny rendent nécessaire l'édition de ce fonds exceptionnel, en particulier la mise à disposition des chercheurs des textes du cartulaire.

Le cartulaire de Reigny n'est pas un chef d'œuvre esthétique, ni un morceau de bravoure littéraire. Son aspect extérieur est des plus miteux : usage du papier, traces d'humidité rendant illisibles certains textes, restaurations contemporaines ayant fait disparaître les cahiers. Son objet est des plus limités : cinquante actes concernant uniquement le domaine abbatial et ses dépendances. C'est un ouvrage destiné à rappeler et à protéger les biens et les droits relevant de la grange de Reigny après les destructions provoquées par le passage des hommes d'armes dans les années 1360. Ce n'est qu'un simple instrument de gestion.

\section{NOTES}

1. M. QUANTIN, Cartulaire général de l'Yonne, t. I, p. 208-210. A.D. Yonne, H 1562, p. 1, acte

1 , copie du comte de Chastellux.

2. M. QUANTIN, Cartulaire général de l'Yonne, p. 300.

3. A.D. Yonne, H 1561 à 1643.

4. Paris, A.N., L 965, n 52.

5. Acquis par la Bibliothèque nationale de France, ce cartulaire a été retrouvé en 1912 par le comte de Chastellux selon le Bulletin de la société des sciences historiques et naturelles de l'Yonne, 66, 3e partie, 1912, séance du 3 mars 1912, p. XXIV. H. STEIN, Bibliographie générale des cartulaires français ou relatifs à l'histoire de France, Paris, 1907, p. 431, n³146. 6. L'intérêt porté aux cartulaires par les historiens n'est pas nouveau, loin s'en faut, mais l'approche est à présent différente. Après avoir été vus comme de simples recueils d'actes, ils sont à présent considérés comme des constructions volontaires et révélatrices d'un projet. A ce sujet, voir les deux colloques organisés à près de dix ans d'intervalle : Les cartulaires, actes de la Table ronde organisée par l'Ecole nationale des chartes et le GDR 121 du CNRS (Paris, 5-7 décembre 1991), réunis par Olivier Guyotjeannin, Laurent Morelle et Michel Parisse, Paris, 1993 ; Les cartulaires méridionau, actes du colloque organisé à Béziers les 20 et $2 \&$ septembre 2002 par le Centre historique de recherches et d'études médiévales sur la méditerranée occidentale (E.A. 3764, Université Paul Valéry - Montpellier III) avec la collaboration du GDR 2513 du 
CNRS (SALVE - Source, acteurs et lieux de la vie religieuse à l'époque médiévale), sous la direction de Daniel Le Blévec, Paris, 2006.

7. La présence de Jean III est attestée entre 1362 et 1391 par les actes. Son successeur, Jean IV Fournier est cité comme abbé de 1391 à 1407. C'est sous un de ces deux abbatiats que le cartulaire a été rédigé.

8. BnF, ms. lat. 17049, fol. 389-400.

9. A.D. Yonne, H 1633, en particulier deux procès-verbaux. Le premier constate en 1666 le passage par force d'un trait de huit chevaux menant huit bateaux en 1666. Le second mentionne en 1725 les dégradations faites au lit de la Cure par le passage des bois flottés de la provision de Paris.

10. De nombreuses chartes ont des pointes de moisissures anciennes.

11. Le comte de Chastellux a eu des difficultés pour lire le manuscrit : il a omis de transcrire les premiers documents et nombre d'actes de sa copie ne sont pas entiers, par choix ou par obligation devant l'état déplorable du papier.

12. En comparant la graphie de ces deux derniers chiffres avec l'écriture de la page de garde, on peut voir des analogies. Le bibliothécaire ayant recensé le document le 27 mai 1870 aurait ajouté les deux numéros manquant.

13. Paris, BnF, ms. lat. 17725 , fol. 30 à 35 .

14. Paris, BnF, ms. lat. 17725, fol. 3v, 4v, 5, 5v, 6, 7, 8, 9, $10 \mathrm{v}, 19$.

15. Par exemple les deux cartulaires de Pontigny, Paris, BnF, ms. lat. 9887 (XII ${ }^{\mathrm{e}}$

$\mathrm{XIII}^{\mathrm{e}}$ siècle) et $\mathrm{BnF}, \mathrm{ms}$. lat. 5465 (XV ${ }^{\mathrm{e}}$ siècle) ou le Grand cartulaire de Clairvaux rédigé au milieu du XIII ${ }^{\mathrm{e}}$ siècle : A.D. Aube, 3 H 9 et 10.

16. Paris, BnF, ms. lat. 17725 , acte 14 , fol. 8 : Omnibus presentes litteras inspecturis, Milo dominus Neoriorum in Domino salutem. Noverint universis quod cum [...] frater Almauricus abbas et conventus Regniacensis et predecessores eorum acquisissent a dominis et heredibus de Tociaco, de sancto Verano, de Baserna et de Argentoil, aquas, feissas et quedam alia in feodo meo absque consensu meo vel predecessorum meorum ut dicebam. Tandem dicti abbas et conventus in hac parte sibi salubriter precanentes petunt predicta acquisita a me sibi confirmari. Ego igitur ad petitionem ipsorum pro remedio anime mee et bone memorie Milonis quondam patris mei necnon et antecessorum et heredum meorum predicto abbati et conventui Regniacensi omnia que ipsi abbas et conventus Regniacensis et predecessores eorum in aquis, feissis, nemoribus, planis et rebus aliis a predictis dominis et heredibus de Tociaco, de sancto Verano, de Baserna et de Argentoil, emptione, elemosina, censiva, commutatione, vel alio quocumque modo vel titulo acquisierunt, tamquam dominus feodi confirmo. Volens et concedens, laudans et approbans quod supra [est, ut] dicti abbas et conventus predicta omnia in perpetuum, pacifice et absolute, possideant et de eisdem pro voluntate sua libere gaudeant et utantur, promisso etiam et ad hoc me obligo quod dictis abbati et conventui Regniacensi predicta omnia et quodlibet illorum adversus omnes homines, bona fide, deliberabo et garentizabo tamquam ea que de feodo meo et de justicia mea. Quod hoc autem omnia firmate, inviolabiliter imperpetuum observenda, obligo me et heredes meos erga dictos abbatem et conventum Regniacenses et constituo [...] in cujus rei testimonium presentes litteras sigilli mei munimine roboravi. Actum anno Domini Millesimo ducentissimo $\mathrm{XL}^{\circ}$ octavo, mense marcio.

17. Paris, BnF, ms. lat. 17725, acte 10, fol. $6 \mathrm{v}:$ « Nous a la supplicacion et request desdicts religieux considérant que en tel cas sommes tenuz de leur donner consoil, confort et aide comme leur gardien, voulons et nous plaist et leur donnons et octroyond de grace especial que ledict fossé et pont leveys, il puissent faire faire devant leur dicte porte, si comme il verront quil leur sera plus necessaire et profitable ». 
18. Ph. CONTAMINE, «L'impact de la guerre de cent ans en France sur le « plat pays » et sur la vie au village ", dans Les villageois face à la guerre (XIV ${ }^{e}$-XVIII ${ }^{e}$ siècle, actes des XXII Journées Internationales d'Histoire de l'abbaye de Flaran, 8-10 septembre 2000, Toulouse, 2002, p. 31.

19. G. GiUliato, «Insécurité et mise en défense du village en Lorraine médiévale », in Les villageois face à la guerre (XIV ${ }^{e}-X V I I{ }^{e}$ siècle, actes des XXII ${ }^{\mathrm{e}}$ Journées Internationales d'Histoire de l'abbaye de Flaran, 8-10 septembre 2000, Toulouse, 2002, p. 36-45.

20. Paris, BnF, ms. lat. 17725 , acte 41 fol. $18-18 \mathrm{v}$ : « Charles, par la grace de Dieu, roy de France. Aus baillis de Sens et Daucerre ou a son lieutenant, salut. Nous biens amés les religieux abbe et convent de leglise de nostre Dame de Reigny de lordre de Cisteaux, nous ont fait expose en complaignant que comme lesdits religieux aient contribue ala closture et fortificacion et emparement de nostre forteresse de Vermenton qui est plus prochain lieu fort de leur eglise ou quel il ont fait faire et edifier ung hostel ou maison a leurs despens, pour mectre et retraire eulz et leurs biens, familiers et serviteurs a sauvete, et toutes foiz quil leur a pleu ou temps passe ont eu retrait et refuge en nostre dicte forteresse, paisiblement et sanz empeschement aucun. Neansmains, Jehan de Lesvres, habitant de la ville de Vermenton soy disant capitaine de ladite forteresse assise en ladite ville ou autres ses lieuxtenants portiers ou commis ala garde dicelle de la volente et commendement dudit Jehan de Lesvres en haine daucuns plais et proces que lesdits religieux pour certaines justes causes ala conservacion du droit de ladite eglise ont et soustiennent touts lesdits habitants de Vermenton ou autrement de leur volente desraisonnable a tort sanz cause et contre raison, ont nagueres refusé et denié ausdits religieux ou a leurs gens, familiers ou serviteurs l'entree de la porte de nostredicte forteresse ». Original conservé : A.D. Yonne, H 1564.

21. Ph. Contamine, op. cit., p. 26. Les moines de Reigny ont également obtenu, en 1371, de la comtesse de Flandres, Marguerite, le droit de retirer leurs biens dans leur maison de Clamecy " pour ce que leurs maisons et habitacions hors forteresses ont esté arses et detruites par les ennemys du Royaume ». A.D. Yonne, H 1583.

22. La région d'Auxerre a été ravagée plusieurs fois dans les années 1360. D’abord en 1358-1360, par les Anglais de Robert Knolles après la défaite de Poitiers. Les Bretons s'installèrent à Arcy-sur-Cure d'où ils rançonnèrent la région. En 1362, les hommes de l'Archiprêtre repoussés d'Alsace par l'empereur Charles IV restèrent en Bourgogne. Enfin, en 1366, une armée royale en route pour la Castille traversa la région. H. DENIFLE, La désolation des Eglises, Monastères et Hôpitaux en France pendant la guerre de Cent Ans, t. I : La guerre de cent ans jusqu'à la mort de Charles V, Paris, 1899 (Impression anastaltique, Bruxelles, 1965), p. 471 et 472. J. FAVIER, La guerre de cent ans, Paris, 1980, p. 265, 307 et 309. N. CÊTRE, op. cit., p. 13 et 22.

23. A.D. Yonne, H 1570, acte de 1369, lignes 9 à 14.

24. A.D. Yonne, H 1570, acte de 1369, lignes 23 à 24.

25. A.D. Yonne, H 1570, acte de 1370. Le problème est que les statuts n'en conservent aucune trace, ni en 1369, ni en 1370, ni même en 1371. En fait, l'explication est donnée par les statuts du chapitre général de 1389 : à cause des désordres enfantés par la guerre, tous les abbés n'ont pu se déplacer pendant longtemps. J. CANIVEZ, Statuta capitulorum généralium ordinis cisterciensis ab anno 1116 ad annum 1786, t. III, 1935, 1369, 1370 et 1370, p. 548-552;1389, i, p. 558 : In primis guerrarum turbines, viarum discrimina et alia quamplurima tempestatum incommoda, quibus hodiernis diebus concutitur et comprimitur, proh dolor! totus mundus, et obedientiae filii a suis sanctis propositis retardantur, considerans madentibus oculis Capitulum generale omnes et singulos abbates Ordinis, qui praesentes 
Capitulo debuerunt personaliter interesse, et nihilominus se super hoc excusantes tenet ipsum Capitulum a via praesentis Capituli favorabiliter excusatos.

26. A.D. Yonne, H 1570, acte de 1371 : ... eisdem abbati et conventui suisque successoribus vendendi tonsuram dicte silve prout melius poterunt, concessimus licenciam et per presentes concedimus per auctoritate predicti capituli generali... : « ... nous avons concédé à l'abbé, à sa communauté et à leurs successeurs la licence de vendre la coupe de la dite forêt au mieux qu'ils pourront, et par la présente nous leur concédons par l'autorité du dit chapitre général... ».

27. A.D. Yonne, H 1578.

28. A.D. Yonne, H 1577.

29. BnF, ms. lat. 17049, p. 389-400.

30. Bulletin de la société des sciences historiques et naturelles de l'Yonne, 66 (1912), p. XXIV.

31. A.D. Yonne, H 1562-2 et 3.

32. Gallia Christiania in provincias ecclesiasticas distributa in qua series et historia archiepiscoporum episcoporum et abbatum regionum omnium quas vetus Gallia, t. XII, Province de Sens et Tonnerre-Auxerre, Paris, 1770. LEBEUF, Mémoires concernant l'histoire civile et ecclésiastique d'Auxerre et de son ancien diocèse, Auxerre, 1848-1855. M. QUANTIN, Cartulaire général de l'Yonne. Recueil de documents authentiques pour servir à l'histoire des pays qui forment ce département, vol. 1, Auxerre, 1854, actes 175, 264, 277, 278, 279, 282, 294, 295, 330,338 et 380 ; vol. 2, Auxerre, 1860, actes 57, 61, 67, 68, 80, 100, 101, 115, 130, 139, 140, $156,157,185,191,197,199,207,219,222,272,289,298,305,313,317,318,339,360,400$, $422,427,438,454,458,474$ et 483 .

33. D. ESCUDIER, « Une charte en langue française de 1164 ? », in BEC, 131 (1973), p. 187-197. L. CAROLUS-BARRE, « Chartes de Guillaume IV, comte de Nevers (1161-1168) pour l'abbaye cistercienne de Reigny : remarques sur « une charte en langue française de 1164 ? » », in BEC, 131 (1973), p. 569-586.

34. D. ESCUDIER, op. cit., p. 196.

35. L. CAROLUS-BARRE, op. cit., p. 570 : « cette charte de 1164, en faveur de l'abbaye de Reigny, dont toutes les données - faits, dates, lieux et personnes cités - paraissent conformes à la vérité, et dont la critique interne ne décèle aucune anomalie grave, présente toutes les apparences d'un acte authentique. Dès lors le clerc qui [...] fit de ce texte une traduction en langue française peut-il être traité de faussaire, et la pièce sortie de sa plume doit-elle être considérée comme un faux?».

36. Nathalie CÊTRE, Etude architecturale de l'abbaye cistercienne de Reigny (Yonne), Mémoire de Maîtrise de l'Université de Bourgogne, 1997.

\section{INDEX}

Mots-clés : abbaye cistercienne, cartulaire

Index géographique : France/Reigny 